Gynäk. Rdsch. 1973;13(Suppl. 1):105

\title{
Wie soll man bei Risikoprolapsen operieren?
}

Diskussionsbemerkung zum 2. Hauptthema

G.

Rieben

Interlaken

Fieberhafter postoperativer Verlauf ist nach vaginaler Hysterektomie im Krankengut des Spitals Interlaken wesentlich häufiger als nach ab-dominalem Vorgehen. Mehrfach kam es zur Bildung subperitonealer Abszesse die sich teils spontan entleerten in Ausnahmefällen eröffnet werden mussten. Eine 62jährige Diabetikerin kam an Douglas- und sub-phrenischem Abszess ad exitum trotz postoperativer Tetrazyklinprophy-laxe. Bei Risikopatientinnen ist es sicherer eine Behelfsoperation durch-zuführen; nur wenn zwingende Gründe vorliegen darf hysterektomiert werden gegebenenfalls kombiniert mit prä- und suboperativer Prophylaxe mit bakteriziden Antibiotika. Jede erst postoperativ gestartete Antibiotika-prophylaxe ist eine Massnahme post contaminationem die zu Recht in Verruf geraten ist. 